\title{
Difficult-to-Treat-Depression-Perceptions of GPs and GP Trainees
}

\author{
Kay M. Jones ${ }^{1}$, Leon Piterman'1, Neil Spike ${ }^{2}$ \\ ${ }^{1}$ Monash University, Melbourne, Australia \\ ${ }^{2}$ Victorian Metropolitan Alliance, Hawthorn, Victoria, Australia \\ Email: ${ }^{*}$ kay.jones@monash.edu
}

Received 8 May 2014; revised 2 June 2014; accepted 27 June 2014

Copyright (C) 2014 by authors and Scientific Research Publishing Inc.

This work is licensed under the Creative Commons Attribution International License (CC BY). http://creativecommons.org/licenses/by/4.0/

(c) (i) Open Access

\section{Abstract}

Introduction: GPs are often the first contact point for mental health concerns. Training to qualify as a GP involves completing a three-to-four year program. Among other things, the training program may include a six-hour mental health training workshop. The study's purpose was to compare GPs' and Trainees' perceptions of difficult-to-treat-depression (DTTD). Methods: Using a semi-structured interview schedule comprising six questions, 10 GPs and six Trainees participated in focus groups or telephone interview. To understand participants' perspectives, data were analyzed using the Framework Method. Findings: Trainees were less clear than GPs about the meaning of DTTD and other diagnostic terms. GPs' diagnosis included querying diagnosis and treatment options. Trainees' experience was limited but they raised barriers including non-response to various treatment trials. Both groups identified management difficulties including: doctor shopping, suicide risk, patients not being well informed; with management difficulties exacerbated by limited access/referring to health professionals, cost and/or unavailability of bulk billing. While some GPs and Trainees had heard of an illness management model or chronic illness model, few used a model. Most reported limited referring to psychiatrists, mainly because of cost and/or limited availability. GPs were more likely to refer to other health professionals and use pharmacological and complementary therapies. Both groups discussed the impact of external factors including cultural factors, patient compliance, treatment failure, and the importance of the relationship between the patient and the professional. Trainees were more likely to stress the importance local clinical guidelines. Discussion and Conclusion: Despite the small size and limited nature, this research provides insight into some of the similarities and differences of GPs' and Trainees' experiences and understanding of DTTD. This may have implications for training providers, clinical supervisors and Trainees, and suggests that enhancement in the role of mental health training may be relevant.

${ }^{*}$ Corresponding author. 


\section{Keywords}

\section{GP Trainees, Difficult-to-Treat-Depression, General Practice Training, Patient Management}

\section{Introduction}

In 2001 the Australian Government introduced the Better Outcomes in Mental Health care (BOMHC) in recognition of GPs' role in managing patients diagnosed with mental health problems [1]. In November 2006, The Better Access to Mental Health Care initiative (Better Access) was introduced [2] in response to low treatment rates for high prevalence mental disorders like depression, and to improve outcomes for people with clinicallydiagnosed problems by providing evidence-based treatment [3] [4]. In July 2009 new Medicare Benefits Schedule (MBS) items were introduced for Mental Health Skills training [5], and the 2011-2012 Australian Government Budget included the introduction of a new time-tiered Medicare items schedule for the preparation of a General Practice Mental Health Treatment Plan (GPMHTP) [6].

Evidence suggests that the overall uptake of the Better Access initiative has been high and has increased over time [3]. In 2007, it was estimated that 3.2 million Australians, or $20 \%$ of the adult population experienced symptoms of a mental disorder [7]; uptake of the initiative increased from one in every 30 in 2007, to one in every 23 in 2008 and to one in every 19 in 2009, and in addition, consumers were generally positive with the model of service delivery and appreciated the clinical care they received [3].

GPs are often the first contact point for mental health concerns [7], particularly since the introduction of the Better Access program [8]. The GPs' focus is on their patient and what can be done in practical terms, for example, for those diagnosed with depression and physical illness [9], chronic pain [10] and cancer [11], rather than focusing on the nuances of classifications [12]. To do this, GPs use a range of options including pharmacological [7] [13], psychosocial [14], and non-pharmacological and/or complementary treatments [15] including diet and exercise [16].

Training to qualify as a GP involves completing a three-to-four year program [17] which is funded by the Australian Government, managed by an independent organisation, General Practice Education and Training Limited (GPET) and delivered across Australia through 17 Regional Training Providers (RTPs) [17] [18]. Among other things, Trainees observe their supervisor [18], consult with patients in general practice [19] and since October 2011, Trainees in the Victorian Metropolitan Alliance (VMA) RTP training program, participate in a structured six-hour mental health training workshop that reflects the content of the BOMHC and Better Access initiatives [20].

One study conducted in Australia found that clinical activities reported as "reasons for encounter" (RFEs per 100 encounters), indicate a trend for Trainees to manage fewer psychological problems (GPRs 6.4 RFE per 100 compared to GPs 7.7RFE per 100) [21]. Possible reasons for differences fall into three broad categories:

1). Patient related, for example, more established and/or older patients may be reluctant for Trainees to manage their chronic/complex conditions [22].

2). “Training program rotation/placement” related, for example, the impact of being time limited (usually 6 months), and/or the impact of geographical location (urban versus rural and/or remote rural posts) on being able to fulfill clinical exposure and/or specific learning goals [19].

3). Training practice specific issues, for example, inadequate teaching time and/or content and structure of teaching, and/or occupational violence [19] [23].

Some issues may be able to be addressed with RTPs providing support [19] [24], others may be addressed by ensuring that current mental health training meets Trainees' needs and requirements [19] [25].

With an aging population and general increase in the prevalence of mental health problems there is a need to ensure that Trainees receive adequate training in the management of chronic disease and mental health.

The aims of this research were to explore any similarities and/or differences between GPs' and Trainees' a) understanding of terms, b) experiences of diagnosis and management and c) knowledge of management options for difficult-to-treat-depression. 


\section{Methods}

\subsection{Sample Recruitment}

In late 2011, a convenience sample of 10 GPs was recruited from inner and outer Melbourne, and rural Victoria through Monash University networks using an email invitation followed by telephone contact. Five GPs (two women and three men) from inner Melbourne participated in a 90-minute face-to-face focus group; three GPs (all men) from outer Melbourne and two GPs (one woman, one man) from rural Victoria participated in a telephone interview of 15 - 30 minutes' duration [12]. In the early months of 2012, a convenience sample of six Trainees (four women, two men) was recruited from VMA (an inner Melbourne RTP), via an email circulated by VMA to all Trainees registered with VMA that year. All had received mental health training via their peer learning workshops prior to the introduction of the structured six-hour mental health training workshop into the VMA training program in October 2012. When Trainees responded and agreed to participate, they provided email contact details to the research team for the purpose of the research team arranging a time, date and venue for the face-to-face interviews [26] [27].

\subsection{Data Collection and Analysis}

A semi-structured interview schedule comprising six headings was developed [12] to address the aims and was used for the focus group and interviews (Table 1). Apart from geographical location and gender, no other demographic data were collected.

The focus groups and interviews were audio-taped and transcribed verbatim by an external organisation.

Data were analysed according to the Framework Method [28] to understand participants' perspectives. Data were analysed manually and independently by investigators (KJ and LP). When there was a difference of opinion, the issues were discussed and agreement reached [28]. Findings, including discussion are reported under the interview schedule's six headings. Comments made by participants are reported as GP focus group (FG 1-5), GP interviews (GP 1-5) [12] and Trainee interviews (TR1-6). Other than those reported here, some results from the GPs’ focus group and interviews have previously been published [12].

Ethics approval to conduct the study was obtained from Monash University Human Research Ethics Committee (MUHREC).

\subsection{Findings}

Findings are reported under the interview schedule’s six sub-headings.

Question 1: What is your understanding of the term difficult-to-treat depression (DTTD)?

There was a variation of understanding in both groups. Some had limited understanding while others demonstrated significant insight. Three inter-related themes emerged from both groups; reference to medication (GP2, GP3, GP4, TR1, TR5, TR6), the patient's response to treatment (GP1, GP5, FG2, TR2, TR3, TR4) and their ability to treat the patient (GP5, FG1, TR3, TR6). Two comments drew the three themes together:

"[DTTD] has not responded to medication and is complicated, long standing, previous history, multiple drug use and recurrence (GP3) ... and is certainly very frustrating” (FG2).

“... meds don't help, overall symptoms aren't improving, or the patient is getting worse, or, get results, then for some reason [the patients] don't take meds then relapse” (TR6).

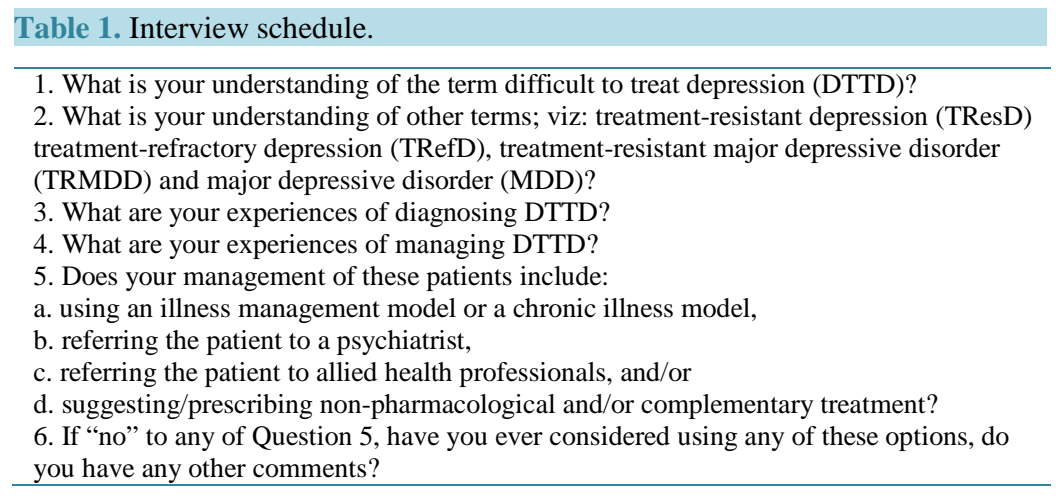


But regardless of the level of understanding and/or defining terminology, all were concerned about helping these patients.

Question 2: What is your understanding of other terms:

- treatment-resistant depression (TResD),

- treatment-refractory depression (TRefD),

- treatment-resistant major depressive disorder (TRMDD), and

- major depressive disorder (MDD)?

Although different words were used, the GPs demonstrated an understanding of the various terms and that these terms generally reflected that several treatment options had been used but depression was still not resolved:

"[treatment resistant depression] is not DTTD and certainly treating them in a situation where life's against them and they're not responding to psychotherapy as well as medication, treatment resistant depression, is often the term I'll get back on discharge summaries from hospitals, or if they've gone to a psychiatrist” (FG5).

One GP suggested that the term DTTD means "it was because the people don't accept they have a problem" (GP4).

Five of the six Trainees recognised the terms but did not understand the specific differences between the terms:

"I don't see any difference between the terms; it's just depression according to DSM IV called major depressive disorder" (TR6).

One Trainee reported having little knowledge or understanding of the terms but similarlyfelt that "... it would be a major depression disorder that fills the DSM IV criteria” (TR2).

Question 3: What are your experiences of diagnosing DTTD?

Not surprisingly, there were differences in GPs' and Trainees' experiences. All GPs had diagnosed patients with DTTD; one GP noted that the percentage of patients is around $10 \%$ in general practice. For the majority, what mattered most was what was happening for the patient, rather than labels which, one GP felt, don’t mean much (GP2). Several GPs initially queried their diagnosis (GP1, GP3, FG1, FG5). Others suggested that part of the diagnostic process included considering whether there were some other methods of treatment (GP1), knowing what medications to use to target different layers of symptoms (GP2) or knowing that not all patients respond to psychiatrists (FG3). For all, confirming the diagnosis was important, and to achieve this, some GPs followed guidelines (GP3, FG5):

"Calling them difficult to treat won't necessarily lead to an obvious next step, so getting clarity about that, that fairly broad category isn't really going to help me to know what to do next with the them [the patients]" (FG2).

The majority of Trainees had limited experience in diagnosing. One Trainee reported having seen previously diagnosed patients who were on antidepressants for some years, but were having relapses of symptoms and mood worsening, leading to the patient feeing worthless thus the Trainee queried both the diagnosis and "the next steps” (TR6). Several described barriers to diagnosing, including non-response to various trials of treatment (TR1, TR3), suggesting:

"You only find out that it's difficult to treat after you've tried treating it" (TR3).

Interestingly, neither GPs nor Trainees mentioned bipolar as distinct from unipolar depression in terms of difficulty with diagnosis [29] [30].

Question 4: What are your experiences of managing DTTD?

GPs reported that they found it difficult to manage patients with DTTD for many reasons or confounders (FG4) such as doctor shopping (GP2), suicide risk (GP2, FG5), patients involved with TAC and/or work cover claims (GP2), patients not being well informed (GP3), and/or not accepting they have a problem (GP4). Several noted that more patients are becoming increasingly difficult to manage (GP3, GP4, FG1) for various reasons including comorbidities, GPs' limited access to services such as psychiatry and primary mental health teams for their patients (FG3, FG4), and understanding the different paradigm for Aboriginal health (FG4). Nonetheless, it was generally agreed that GPs have an important role to play and that they are good position to make a holistic assessment because they know about the physical and family issues.

Not surprisingly, most Trainees had limited practical experience in managing DTTD, although they understood the problems and pitfalls. Some identified several management issues with similarities to those mentioned by the GPs, while others identified few. Suicide risk (TR1, TR2), relapse (TR4), accessing and/or referring to 
other health professionals such as psychiatrists (TR3, TR4, TR5), stigma (TR6) and psychosocial issues including lack of funds and family support (TR3, TR6) were identified as challenges when managing patients diagnosed with DTTD. Of importance was ensuring there is a safety net for the patient (TR1, TR5), establishing a treatment plan (TR2), taking a holistic approach and looking at all factors (TR2), including other diagnoses (TR3, TR6), providing patient education (TR6) and information about how to access help such as the hospital psychiatrist, Lifeline, or to call a friend (TR5). Others identified difficulties such as:

"... some patients not really fitting into a box; management is never really clean cut because other things such as drug use, social problems or financial difficulties can affect the patient" (TR3).

In addition, cost and unavailability of bulk billing were described as barriers for some patients (TR6) and to access other health professionals including psychiatrists for others (TR3, TR5):

"The main thing is to make sure that they're actually getting optimum treatment" (TR1).

"If they're feeling suicidal or particularly depressed, then establishing a treatment plan, whether or not that might include medication" (TR2).

"It's very difficult first of all to get put through to a psychiatrist and speak with them, andsecond of all to try and get an appointment" (TR5).

Question 5: Does your management of these patients include:

a). using an illness management model or chronic illness model,

b). referring to a psychiatrist,

c). referring to allied health professionals and/or

d). suggesting/prescribing non pharmacological and/or complementary treatment?

a). Using an illness management model.

One GP had heard ofand used the Wagner model [31]. Around half had heard of models but not used them, the others had not heard of, or used models:

"Yes, Wagner, and I find it useful" (GP5).

Only two Trainees knew what an illness management model or a chronic illness model was, but had not used them (TR3, TR5). One described a "mental health plan" as an illness management model:

"Yeah, so I've got them on the mental health plan" (TR1).

"It would be more a chronic illness plan" (TR4).

\section{b). Referring to a psychiatrist}

Different approaches were noted in the referral patterns to psychiatrists. Most GPs reported they rarely referred to a psychiatrist for several reasons; cost and availability (GP1, GP2), unavailability of bulk billing (GPs), and great difficulty in accessing services via, for example, a community health service with a mental health facility (GP4, GP5). Nonetheless, the professional relationship between the GP and psychiatrist was described as important (GP2).

"Availability is more an issue than cost" (GP1).

"I think I would like more access to primary mental health teams, especially given that the public psychiatry is often difficult to access" (FG3).

In comparison, all Trainees indicated they would refer to a psychiatrist after trying drugs (TR5) and a psychologist (TR4, TR5), but referring depended on the patient and the situation (TR4, TR6) because some people may be resistant to engaging with, for example, a psychologist (TR2). Other options included referring to another GP for a second opinion or a consult (TR1, TR3), referring to a psychiatrist if the patient had previously been seen by a psychologist (TR3, TR4), calling the "on call” psychiatry registrar (TR5), or asking for help from colleagues (TR6). None mentioned cost or availability as barriers to accessing services which may reflect lack of practical experience:

"If I thought they [the patients] were more at risk of harming themselves, I'd refer to public or private; I'd write a referral letter, fax it through and find out their availability [psychiatrists], but in the interim I'd call up the psychiatry registrar on call" (TR5).

"I'd be asking for help from my colleagues and then it would be a matter of referring to either a psychiatrist or psychologist for extra help" (TR6).

\section{c). Referring to allied health professionals}

Different patterns of referring emerged. All GPs referred to allied health professional but the majority of Trainees had not; whether this was because of lack of opportunity or lack of knowledge of the process was not discussed. 
GPs referred to allied health professionals because availability was broader and the cost more affordable for the majority of patients (GP1, GP2). However, while an increase in the use of mental health care plans (MHCPs) by GPs has been noted, GPs reported that external events have had an impact; for example the Australian Government announced, that from 2012, a reduction in the number of Medicare funded visits to psychologists from 12 to 10 during the calendar year (GP3).

"For patients who have moderate or severe depressive illness, if there is willingness on the patients' part, and there is some indication that the patient would respond to focussed psychological strategies [would refer to allied health professionals]" (GP5).

While some GPs reported a noticeable reduction in the availability of counsellors, psychologists and social workers; one raised concerns of some allied health professionals' methods:

"I'm not entirely thrilled with some of their methods of treatment; once you go there you seem to be going forever. One I referred to recently, wanted me to ring and get a special authority to have a longer consultation with them, I wasn't very impressed with that. So, because the patients don't pay it seems like a bad system. If patients pay they put more value to the service.... I don't refer patients who are depressed to allied health professionals like social workers or occupational therapists" (GP4).

The majority of Trainees felt that referring to allied health professionals would depend on various issues such as the patient's situation (TR4), including whether the patient was homeless (TR5), had unusual family dynamics such as domestic violence (TR5), cultural aspects (TR2), and/or co-morbidities (TR3) such as pain and/or disability (TR2). One Trainee felt there was no need to refer to allied health professionals (TR6). However, financial issues (TR1) and the limited availability of financial assistance via Medicare items such as general practice management plans (GPMPs) and mental health care plans (MHCPs) were described as concerns (TR1):

"If they're on a mental health care plan and they don't have another chronic health condition, then they're actually-you can't actually do a GP management plan for them, which I find a bit discriminatory for people with chronic mental health conditions" (TR1).

"Depression is such a multi-factorial thing-which certainly allied health practitioners could be extremely useful for it, I have done-referred a lot to in the past" (TR2).

"Routinely, I don't see how allied health could help" (TR5).

“I haven't needed to so, no, I haven't done that” (TR6).

d). Suggesting/prescribing non pharmacological and/or complementary treatment

While all GPs had used non-pharmacological and/or complementary treatments, Trainees’ experience and opinion varied.

All GPs reported they were aware of their patients' use of non-pharmacological and/or complementary treatments, whether the GP suggested, or patients independently explored options. Regardless of the many and varied options mentioned and used, general consensus was that the value of the patient's compliance with a treatment plan was vital. Options included swimming (GP2), yoga, Pilates (GP3) relaxation and meditation (GP4, FG1, FG4), rebirthing as a relaxation technique (FG2), exercise and morning sunshine (FG4) hypnotherapy, acupuncture, sleep hygiene, setting an agenda for the day (FG5) chiropractor, spiritual therapist (FG1), focussed psychological strategies (GP5), melatonin for sleep disturbance (GP2, FG1), St John’s Wort (GP2, GP3, FG5), and nutrition advice (GP2). Also noted was the importance of reducing smoking (FG1) and cannabis use (FG1, FG4).

"Often the patients will have tried that themselves or would have had advice from other sources to try that but I don't use that as a primary treatment option" (GP1).

"Swimming, yeah; my theory is three hours of exercise a week is equivalent of an SSRI every day in its effect on mood. That's what makes me so furious that we have a system that pays for the patch up jobs but will not in any way subsidise something that's much more beneficial all round" (GP2).

"I say the only way to come off medication is if you resort to alternative methods of collecting your thoughts and relaxing and if you really want to get off medication here's what you need to do. I do that but that's about it" (GP4).

Trainees' opinions and experience were more limited but varied; not everyone had suggested complementary treatment (TR6); some felt they were not sufficiently knowledgeable on the subject (TR3, TR4). General consensus was that what could be considered as holistic and/or whether health insurance providers covered options such as dietician and physiotherapy needed to be clarified (TR1). While there was support for patients' decisions (TR1), others felt it was more dependent on the patient's needs or as first-line for mild-to-moderate depression, 
but not something as first line treatment for major depression (TR2, TR4, TR5). Mindfulness practiced by psychologists and GPs was described as positive particularly because of connectedness to someone in the community; there was also a place in management plans when, for example, dealing with sleep issues (TR2, TR4), developing a good diet and exercise plan (TR1, TR3), for coping techniques (TR5) or for relaxation strategies (TR6).

Some felt these options may be problematic for a number of reasons, for example, some complementary medicines may be "addictive" (TR2) or there was insufficient evidence for some complementary medicines but increasing evidence for others, thus it is incumbent on the practitioners to be knowledgeable about them (TR5). Regardless, it was important to check whether the patients were already using anything (TR3):

"Maybe St John's Wort, well ask them if they are already taking anything like that, just because obviously there can be interactions and side effects if they're taking those together" (TR3).

"If the patient is complaining about sleeping problems, then possibly valerian as atrial with the sleep" (TR4).

"I'm not convinced there's enough evidence on it, and I wouldn't want to start something that messes up someone's mood, and then, you know ... if a patient said that they wanted to go see their own complementary and alternative medicine practitioner, I wouldn't stop them, but I wouldn't encourage it, or tell them to go" (TR5).

Question 6: If "no" to any of Question 5, have you ever considered using any of those options, do you have any other comments?

GPs raised more, and more varied issues than the Trainees. One GP talked about the difficulty of the management cycle, particularly the cyclic changes that patients may have and the episodic crises that have to be dealt with as an emergency (GP1). Another raised the use and value of guidelines (GP3). The impact of external factors were also important to take into consideration, for example, cultural factors (FG1), patient compliance, treatment failure, the importance of the relationship between the patient and the professional (FG5) and engaging the patient in the decision making process (FG1, FG3) even when they are so profoundly depressed that any choice is way beyond them (FG1, FG2). Patients accessing information via the internet was noted as relatively new phenomenon which also needed to be taken into account (FG5):

“... and cultural factors; it was interesting. She believed that the baby doesn't want her to have that medicine- 'that's why I'm vomiting"” (FG1).

"Sometimes the people who are difficult to treat become the heart sink patient who you just don't really; the whole relationship becomes a bit dysfunctional and they, may be eventually they leave or you refer them out somewhere else" (FG3).

"There's been a shift in the modus operandi isn't there in the model of care?" (FG4).

"Patients have consulted the third doctor, Doctor Google and they come in sort of a bit more informed or I think the second visit after you've discussed the medication, discussed the research, and talk to you about it and it becomes more a business partnership rather than doctor patient sort of thing you sort of trying to be a team member to try and be themselves, they'll have input, you'll have input, at the end of the day they understand you are both on the same wavelength" (FG5).

Trainees made additional comments the importance of more research, their limited exposure during training, and the value of guidelines:

"The importance of more work being done on depression" (TR2, TR5).

"As DTTD is a chronic disease, and Trainees only see patients over a short time during their training placements, their exposure to DTTD is limited, we're really only seeing a small amount of that because we're not seeing patients over a long term and because depression, you know, takes a while" (TR2).

"Guidelines are important, particularly if they are available on the internet to assist with a more step-wise approach" (TR6).

\section{Discussion and Conclusion}

This research provides insight into some of the similarities and differences of GPs' and Trainees' understanding of terms, experiences of diagnosis, management and knowledge of management options.

Not surprisingly, GPs had a deeper understanding of terms than the Trainees although both felt the terms were not of practical importance. Trainees demonstrated less knowledge about availability and value of chronic illness models [31], government initiatives [1] [4]-[6] and positive feedback from patients [3]. Nonetheless, there 
was general consensus that the GP is generally the patients' first contact point for their mental health concerns [7] including depression [8], particularly when management included physical illnesses [9], chronic pain [10] and/or cancer [11], as they commonly occur with major depression with a complex association between the two [9]-[11].

All GPs and some Trainees, reported the importance and value of using a range of treatments and management options including pharmacological [13], psychosocial [14], non-pharmacological and/or complementary treatment [15] including diet and exercise [16]; and were aware that some options may not be available for some patients because of limited personal resources and/or Medicare funding requirements [6].

GPs had significantly more experience than Trainees in diagnosing and managing patients with DTTD. As Trainees' placements/rotations are time-limited [23], they may benefit with more time to focus on diagnosing and managing patients with chronic disease, particularly patients with mental illness. The program undertaken by this cohort of Trainees, which is underpinned by adult learning models and the principles of experiential, needs focussed, purpose driven learning and quality feedback [17] [18] [20], appears to have equipped them with the knowledge to manage depression but not the experience to translate this into practice [19]. However, some Trainees may benefit with additional experience of participating in a structured attachment where they can develop mental health management plans and thus gain a better understanding of what is involved, particularly when other chronic diseases are involved [9] [10]. Although not directly questioned, the impact of patients' age 22, training issues [19] [25], including supervision [19] [23] and/or personal wellbeing [19] [24], were raised as matters of concern to Trainees.

The generalisability of this study may be limited because of the small number of participants, the majority of whom were from metropolitan Melbourne, with only two from the rural sector.

Nonetheless, this study contributes to understanding the value of Trainees' training experiences and needs, provides insight into Trainees experiences not previously reported, and raises suggestions for generally improving diagnosis and management of DTTD [12].

\section{Issues for Training Providers}

- The need for more clearly defined experiences and learning opportunities as Trainees progress through the stages of training,

- Better identifying the training practices that provide various levels of exposure/experience in managing patients with major depression,

- More accurate data on the range of patients seen by Trainees and the use of various treatment modalities, for example, the ReCEnT data collection [19],

- Greater access to practices/facilities with a focus on mental health/illness for special skills training terms,

- Better understanding of the role/s of allied health professionals, team based care and mental health care planning, and the application of these in general practice during training placement/rotations,

- With increasing numbers of international medical graduates in GP training, language and culture in the management of mental health/illness in general practice need to be considered, as they add complexity to an already complex issue.

\section{Acknowledgements}

The authors sincerely thank the GPs and Trainees who participated in the focus groups and/or interviews.

This project was funded by a grant from AstraZeneca.

\section{References}

[1] Australian Government DoHaA: Programs. Better Access to Mental Health Care. In: Canberra, 2010. http://www.health.gov.au/internet/main/publishing.nsf/Content/mental-ba-eval-ques

[2] Australian Government Department of Health and Ageing: Better Access to Mental Health Care-Questions and Answers. In: Canberra, 2006. www.health.gov.au/internet/main/publishing.nsf/Content/coag-mental-q\&a.htm

[3] Pirkis, J., Harris, M., Hall, W. and Ftanou, M. (2011) Evaluation of the Better Access to Psychiatrists, Psychologists and General Practitioners through the Medicare Benefits Schedule Initiative. Summative Evaluation, Final Report. 
Centre for Health Policy, Programs and Economics, The University of Melbourne.

[4] Australian Government DoHaA: Fact Sheet. Key findings from the program evaluation of the Better Access to Psychiatrists, Psychologists and General Practitioners through the Medicare Benefits Schedule initiative. Australian Government, 2011. http://www.health.gov.au/internet/main/publishing.nsf/Content/mental-ba-eval-fact

[5] Australian Government DoHaA: Fact Sheet. Implementation of Mental Health skills Training for General Practitioners: 2009-10 Budget Measure. Australian Government. 2009. http://www.health.gov.au/internet/main/publishing.nsf/Content/mental-ba-train

[6] Australian Government DoHaA: Fact Sheet. New Medicare Items for GP Mental Health Treatment Plans: 2011-12 Budget Measure. Australian Governemnt, 2011. http://www.health.gov.au/internet/main/publishing.nsf/Content/mental-ba-fact-gp

[7] AIHW: Mental health services in Australia 2007-08 (Cat.no.HSE 88). In: Canberra: AIHW; 2010.

[8] Harrison, C.M., Britt, H.C. and Charles, J. (2012) Better Outcomes or Better Access—Which Was Better for Mental Health Care? MJA, 197, 170-172.

[9] Olver, J.S. and Hopwood, M.J. (2012) Depression and Physical Illness. MJA Open, 1, Suppl. 4, 9-12.

[10] Holmes, A., Christelis, N. and Arnold, C. (2012) Depression and Chronic Pain. MJA Open, 1, Suppl. 4, 17-20.

[11] Couper, J.W., Pollard, A.C. and Clifton, D.A. (2012) Depression and Cancer. MJA Open, 1, Suppl. 4, 13-16.

[12] Jones, K.M., Castle, D.J. and Piterman, L. (2012) Difficult-to-Treat-Depression: What Do General Practitioners Think? MJA, MJA Open, 1, Suppl. 4, 6-8.

[13] Chan, H.-N., Mitchelle, P.B., Loo, C.K.A. and Harvey, S.B. (2012) Pharmacological Treatment Approaches to Difficult-to-Treat Depression. MJA Open, 1, Suppl. 4, 44-47.

[14] Casey, M.F., Perera, D.N., Clarke, D.M. (2012) Psychosocial Treatment Approaches to Difficult-to-Treat Depression. MJA, MJA Open, 1, Suppl. 4, 52-55.

[15] American Psychiatric Association: Practice Guideline for the Treatment of Patients with Major Depressive Disorder. 2010. http://www.psych.org/guidelines/mdd2010

[16] Jacka, F.N. and Berk, M. (2012) Depression, Diet and Exercise. MJA Open, 1, Suppl. 4, 21-23.

[17] General Practice Education and Training Limited (GPET) (2013) Australian General Practice Training Handbook 2014. GPET. http://www.agpt.com.au

[18] Longman, C. and Temple-Smith, M. (2013) General Practice Registrar Observation of Their Supervisors in Consultation. AFP, 42, 418-421.

[19] Morgan, S., Magin, P.J., Henderson, K.M., Goode, S.M., Scott, J., Bowe, S.J., Regan, C.M., Sweeney, K.P., Jackel, J. and van Driel, M.L. (2012) Study Protocol: The Registrar Clinical Encounters in Training (ReCEnT) Study. BMC Family Practice, 13, 50. http://www.biomedcentral.com/1471-2296/13/50

[20] Johnson, C. and Spike, N. (2011) Mental Health Skills Training for GP Registrars. In: Victorian Metropolitan Alliance (Unpublished).

[21] Spike, N. and Britt, H. (2005) The Clinical Activities of VMA Registrars in Each Stage of Training. In: Victorian Metropolitan Alliance, Unpublished, Victorian Metropolitan Alliance, Melbourne.

[22] Bonney, A., Jones, S.C., Phillipson, L. and Iverson, D. (2010) General Practice Registrars Attitudes of Older Patients. AFP, 39, 419-424.

[23] Larkins, S.L., Spillman, M., Parison, J., Hays, R.B., Vanlint, J. and Veitch, C. (2004) Isolation, Flexibility and Change in Vocational Training for General Practice: Personal and Educational Problems Experienced by General Practice Registrars in Australia. Family Practice, 21, 559-566. http://dx.doi.org/10.1093/fampra/cmh513

[24] Schattner, P., Mazalin, D., Pier, C., Wainer, J. and Ling, M.Y. (2010) GP Registrar Well-Being: A Cross-Sectional Survey. Asia Pacific Family Medicine, 9, 2. http://www.apfmj.com/content/9/1/2

[25] Ratcliffe, J., Gask, L., Creed, F. and Lewis, B. (1999) Psychiatric Training for Family Doctors: What Do GP Registrars Want and Can a Brief Course Provide This? Medical Education, 33, 434-438. http://dx.doi.org/10.1046/j.1365-2923.1999.00343.x

[26] Liamputtong, P. and Ezzey, D. (2005) Qualitative Research Methods. Oxford University Press, Melbourne.

[27] Polgar, S. and Thomas, S. (2005) Introduction to Research in the Health Sciences. Elsevier Churchill Livingstone, Sydney.

[28] Ritchie, J. and Spencer, L. (1994) Qualitative Data Analysis for Applied Policy Research. In: Bryman, A. and Burgess, B., Eds., Analyzing Qualitative Data, Routledge, London.

[29] Castle, D.J., Berk, M. and Hocking, B.M. (Eds.) (2010) Bipolar Disorder: New Understandings, Emerging Treatments. 
MJA, 193, S1-S40.

[30] Piterman, L., Jones, K.M. and Castle, D.J. (2010) Bipolar Disorder in General Practice: Challenges and Opportunities. MJA, 193, S14-S17.

[31] Wagner, E.H. (1998) Chronic Disease Management: What Will It Take to Improve Care for Chronic Illness? Effective Clinical Practice, 1, 2-4. 
Scientific Research Publishing (SCIRP) is one of the largest Open Access journal publishers. It is currently publishing more than 200 open access, online, peer-reviewed journals covering a wide range of academic disciplines. SCIRP serves the worldwide academic communities and contributes to the progress and application of science with its publication.

Other selected journals from SCIRP are listed as below. Submit your manuscript to us via either submit@scirp.org or Online Submission Portal.
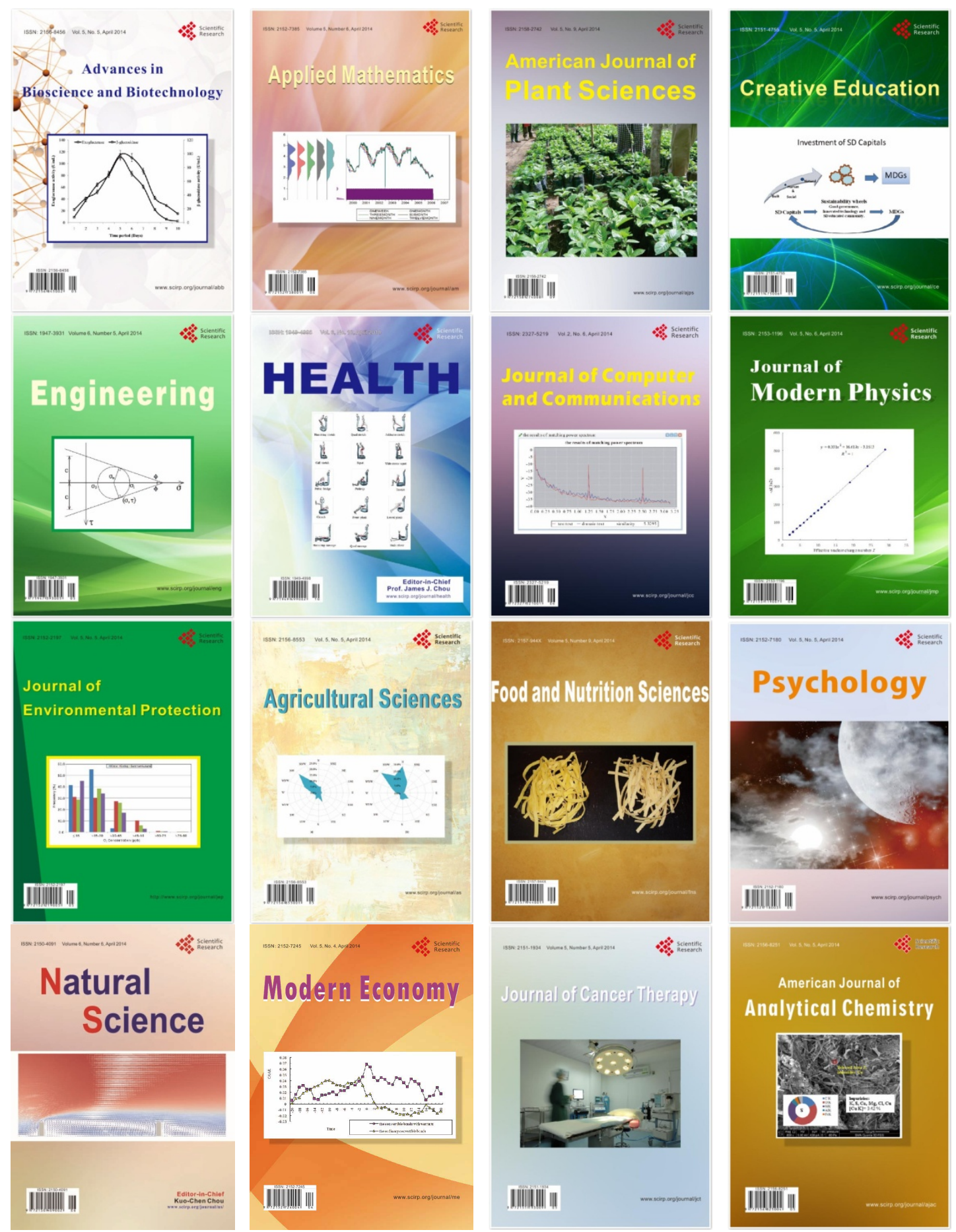